\title{
ACCELERATED SPECTRAL REFINEMENT PART I: SIMPLE EIGENVALUE
}

\author{
RAFIKUL ALAM ${ }^{1}$, REKHA P. KULKARNI ${ }^{1}$ and BALMOHAN V. LIMAYE ${ }^{1}$
}

(Received 27 November 1995; revised 11 June 1998)

\begin{abstract}
A general framework is developed for constructing higher order spectral refinement schemes for a simple eigenvalue. Well-known techniques for ordinary spectral refinement are carried over to higher order spectral refinement yielding faster rates of convergence. Numerical examples are given by considering an integral operator.
\end{abstract}

\section{Introduction}

Spectral refinement is a procedure that allows one to approximate eigenelements of a very large discrete system by successively improving upon the eigenelements obtained from a coarse model through direct methods. Several refinement methods such as the Rayleigh-Schrödinger method $([2,7,8,14,15])$, the fixed slope Newton scheme and its variants $([3,4,8,11,15])$ and the defect correction method $([1,8,16])$ have been studied for approximating a simple eigenvalue. These methods avoid solving large matrix eigenvalue problems, thus saving time and memory. However, the convergence of these methods can be very slow, and to achieve a desired accuracy it may even be necessary to consider a finer discretization, which often results in computational complexities. In [9] Dellwo proposed two higher order refinement schemes for a simple eigenvalue which achieve better rates of convergence. His schemes involve the solution of a polynomial eigenvalue problem of order $q \geq 1$. While his first scheme reduces to the well-known fixed slope Newton scheme when $q=1$, his second scheme is obtained from the first by simply adding an extra term in the summations.

The main objective of this paper is to develop a general framework for constructing a higher order spectral refinement for a simple eigenvalue. Let $\left(T_{n}\right)$ be a sequence of bounded operators on a complex Banach space $X$ approximating a bounded operator

\footnotetext{
'Department of Mathematics, Indian Institute of Technology, Bombay 400 076, India.

(C) Australian Mathematical Society 2000, Serial-fee code 0334-2700/00
} 
$T$ in the following sense: the sequence $\left(\left\|T_{n}\right\|\right)$ is bounded and $\left\|\left(T-T_{n}\right)^{2}\right\| \rightarrow 0$ as $n \rightarrow \infty$. We construct two operators $\mathbf{T}_{q}^{(n)}$ and $\mathbf{T}_{q, n}$ on the product space $X_{q}$, consisting of $q$ copies of $X$, which have the properties:

(i) If $\lambda \neq 0$, then $\lambda \in \sigma(T)$ if and only if $\lambda \in \sigma\left(\mathbf{T}_{q}^{(n)}\right)$ and the algebraic multiplicity of $\lambda$ as an eigenvalue of $T$ is the same as the algebraic multiplicity of $\lambda$ as an eigenvalue of $\mathbf{T}_{q}^{(n)}$;

(ii) $\left\|\mathbf{T}_{q, n}-\mathbf{T}_{q}^{(n)}\right\|_{\infty}=\left\|\left(T-T_{n}\right)^{q}\right\|$;

(iii) $\lambda_{q, n}$ is an eigenvalue of $\mathbf{T}_{q, n}$ and $\mathbf{T}_{q, n} \Phi_{q, n}=\lambda_{q, n} \Phi_{q, n}$ for some nonzero $\Phi_{q, n}$ in $\mathbf{X}_{q}$ if and only if $\Phi_{q, n}=\left[\phi_{q, n}, \frac{\phi_{q, n}}{\lambda_{q, n}}, \ldots, \frac{\phi_{q, n}}{\left(\lambda_{q, n}\right)^{q-1}}\right]^{t}$, where the first component $\phi_{q, n}$ of $\Phi_{q, n}$ satisfies

$$
\left(\left(\lambda_{q, n}\right)^{q} I-\sum_{j=0}^{q-1}\left(\lambda_{q, n}\right)^{q-1-j} \Delta_{n}^{j} T_{n}\right) \phi_{q, n}=0 .
$$

The last equation states the polynomial eigenvalue problem considered by Dellwo in [9]. Several well-known refinement schemes for a simple eigenvalue can be upgraded to higher order schemes by considering the operators $\mathbf{T}_{q}^{(n)}$ and $\mathbf{T}_{q, n}$. Although the proofs of the error estimates for these accelerated refinement schemes are similar to the case when $q=1$, one needs to show that various constants appearing in the error bounds are independent not only of $n$ but also of the order $q$. Then one can conclude that a larger value of $q$ would imply a faster rate of convergence of the iterates. This approach is illustrated by considering the fixed slope Newton scheme. We first prove the results under the additional assumption $|\lambda|>1$. Then the results are proved in general by scaling all the operators involved in the process. The scaling factor is based on the knowledge of a lower bound (not necessarily sharp) for $|\lambda|$. The implementability of the accelerated refinement schemes is also discussed. Numerical examples for computing the largest and the second largest simple eigenvalue of an integral operator, which was considered by Dellwo, are presented. Our results compare favourably with those given by him in [9].

An additional merit of our approach is that it can be employed to treat the case of a multiple eigenvalue or, more generally, of a cluster of eigenvalues whose total algebraic multiplicity is finite. This will be considered in Part II of this paper. It is not clear how Dellwo's schemes given in [9] can be extended to treat this general case.

\section{Preliminaries}

Let $X$ be a complex Banach space and $B L(X)$ denote the Banach space of all bounded linear operators on $X$ along with the operator norm. For $T \in B L(X)$, let 
$\rho(T)$ and $\sigma(T)$ denote the resolvent set and the spectrum of $T$, respectively:

$$
\rho(T)=\left\{z \in \mathbb{C}:(T-z I)^{-1} \in B L(X)\right\} \quad \text { and } \quad \sigma(T)=\mathbb{C} \backslash \rho(T) .
$$

The spectral radius of $T$ is defined as

$$
r_{\sigma}(T)=\sup \{|\lambda|: \lambda \in \sigma(T)\} .
$$

Unless otherwise stated, $\Gamma$ will denote a simple closed positively oriented rectifiable curve in $\mathbb{C}$ and $\ell(\Gamma)$ will denote its length.

We are interested in numerically solving the eigenvalue problem

$$
T \phi=\lambda \phi, \quad \lambda \in \mathbb{C}, \quad 0 \neq \phi \in X .
$$

Let $\left(T_{n}\right)$ be a sequence of operators in $B L(X)$ and $\Delta_{n}=T-T_{n}$. In [9], Dellwo considered the following polynomial eigenvalue problem which is supposed to provide an approximate solution of the eigenvalue problem for $T$ :

$$
\left(\left(\lambda_{q, n}\right)^{q} I-\sum_{j=0}^{q-1}\left(\lambda_{q, n}\right)^{q-1-j} \Delta_{n}^{j} T_{n}\right) \phi_{q, n}=0, \quad \lambda_{q, n} \in \mathbb{C}, \quad 0 \neq \phi_{q, n} \in X .
$$

Notice that for $q=1$ the polynomial eigenvalue problem reduces to

$$
T_{n} \phi_{1, n}=\lambda_{1, n} \phi_{1, n}, \quad 0 \neq \phi_{1, n} \in X .
$$

We show that the polynomial eigenvalue problem can be converted to the ordinary eigenvalue problem defined on a product space. For this purpose we introduce the following notations. Let $q$ be a positive integer and $\mathbf{X}_{q}$ denote the set of all column vectors $\mathbf{x}=\left[x_{1}, \ldots, x_{q}\right]^{t}$ with $x_{1}, \ldots, x_{q}$ in $X$. Define

$$
\|\mathbf{x}\|_{\infty}=\max \left\{\left\|x_{j}\right\|: j=1, \ldots, q\right\} .
$$

Then $\mathrm{X}_{q}$ is a Banach space with respect to the norm \|\|$_{\infty}$. We identify the adjoint space of $\mathbf{X}_{q}$ with the set of all column vectors $\mathbf{x}^{*}=\left[x_{1}^{*}, \ldots, x_{q}^{*}\right]^{t}$ with $x_{1}^{*}, \ldots, x_{q}^{*}$ in $X^{*}$. Define

$$
\left\|\mathbf{x}^{*}\right\|_{1}=\left\|x_{1}^{*}\right\|+\cdots+\left\|x_{q}^{*}\right\| .
$$

If we let

$$
\left\langle\mathbf{x}, \mathbf{x}^{*}\right\rangle=\left\langle x_{1}, x_{1}^{*}\right\rangle+\cdots+\left\langle x_{q}, x_{q}^{*}\right\rangle,
$$

then it is clear that $\left|\left(\mathbf{x}, \mathbf{x}^{*}\right\rangle\right| \leq\|\mathbf{x}\|_{\infty}\left\|\mathbf{x}^{*}\right\|_{1}$. 
For nonzero $z$ in $\mathbb{C}$, let

$$
A_{q, n}(z)=z I-\sum_{j=0}^{q-1} \frac{\Delta_{n}^{j} T_{n}}{z^{j}} .
$$

The polynomial eigenvalue problem can be written as

$$
\lambda_{q, n}^{q-1} A_{q, n}\left(\lambda_{q, n}\right) \phi_{q, n}=0, \quad 0 \neq \phi_{q, n} \in X .
$$

Let $\mathbf{T}_{q, n}$ be the following companion matrix of the polynomial $z^{q-1} A_{q, n}(z)$ :

$$
\left[\begin{array}{ccccc}
T_{n} & \Delta_{n} T_{n} & \cdots & \cdots & \Delta_{n}^{q-1} T_{n} \\
I & 0 & \cdots & \cdots & 0 \\
0 & \ddots & \ddots & & \vdots \\
\vdots & & \ddots & \ddots & \vdots \\
0 & 0 & \cdots & I & 0
\end{array}\right]
$$

If $\lambda_{q, n} \neq 0$, then

$$
\mathbf{T}_{q, n} \Phi_{q, n}=\lambda_{q, n} \Phi_{q, n}, \quad \Phi_{q, n} \in \mathbf{X}_{q}
$$

if and only if $\Phi_{q, n}=\left[\phi_{q, n}, \frac{\phi_{q, n}}{\lambda_{q, n}}, \ldots, \frac{\phi_{q, n}}{\left(\lambda_{q, n}\right)^{q-1}}\right]^{t}$, where the first component $\phi_{q, n}$ of $\Phi_{q, n}$ satisfies

$$
A_{q, n}\left(\lambda_{q, n}\right) \phi_{q, n}=0 .
$$

For the rest of this paper, we let $q \geq 2$ and make the hypothesis:

(H): $\left(\left\|T_{n}\right\|\right)$ is a bounded sequence and $\left\|\left(T-T_{n}\right)^{2}\right\| \rightarrow 0$ as $n \rightarrow \infty$.

Then for all large $n$, we have

$$
\left\|\mathbf{T}_{q, n}\right\|_{\infty} \leq \max \left\{1,2\left(\left\|T_{n}\right\|+\left\|\Delta_{n} T_{n}\right\|\right)\right\} .
$$

Let $\Lambda$ be a cluster of nonzero eigenvalues of $T$ whose total algebraic multiplicity is finite, say $m$, and which is isolated by a curve $\Gamma$ in $\rho(T) \backslash\{0\}$ from the rest of $\sigma(T)$ as well as from 0 , that is, $0 \notin \Gamma \cup \operatorname{Int} \Gamma$ and $\sigma(T) \cap \operatorname{Int} \Gamma=\Lambda$.

The following results were proved in Proposition 3.2 (b) and Theorem 3.5 of [6].

PROPOSITION 2.1. (a) For all large $n$ and all $q=2,3, \ldots, \Gamma \subset \rho\left(\mathbf{T}_{q, n}\right)$, so that

$$
\mathbf{P}_{q, n}=-\frac{1}{2 \pi i} \int_{\Gamma}\left(\mathbf{T}_{q, n}-z \mathbf{I}_{q}\right)^{-1} d z
$$

where $\mathbf{I}_{q}$ is the identity operator on $\mathbf{X}_{q}$, defines the spectral projection associated with $\mathbf{T}_{q, n}$ and $\Lambda_{q, n}=\sigma\left(\mathbf{T}_{q, n}\right) \cap \operatorname{Int} \Gamma$, and

$$
\operatorname{rank} \mathbf{P}_{q, n}=m .
$$


(b) If $\min \{|z|: z \in \Gamma\}>1$, then for all large $n$ and all $q=2,3, \ldots$,

$$
\max _{z \in \Gamma}\left\|\left(\mathbf{T}_{q, n}-z \mathbf{I}_{q}\right)^{-1}\right\|_{\infty} \leq C
$$

where $C$ is a constant independent of $q$ and $n$.

We now introduce another operator $\mathbf{T}_{q}^{(n)}: \mathbf{X}_{q} \rightarrow \mathbf{X}_{q}$ such that for $\lambda \neq 0, \lambda \in \sigma(T)$ if and only if $\lambda \in \sigma\left(\mathbf{T}_{q}^{(n)}\right)$, the algebraic multiplicity of $\lambda$ as an eigenvalue of $T$ is the same as the algebraic multiplicity of $\lambda$ as an eigenvalue of $\mathbf{T}_{q}^{(n)}$, and $\left\|\mathbf{T}_{q, n}-\mathbf{T}_{q}^{(n)}\right\|_{\infty}=$ $\left\|\left(T-T_{n}\right)^{q}\right\|$.

For nonzero $z$ in $\mathbb{C}$, let

$$
A_{q}^{(n)}(z)=z I-\sum_{j=0}^{q-2} \frac{\Delta_{n}^{j} T_{n}}{z^{j}}-\frac{\Delta_{n}^{q-1} T}{z^{q-1}} .
$$

It is easy to see that

$$
A_{q}^{(n)}(z)=\sum_{j=0}^{q-1} \frac{\Delta_{n}^{j}}{z^{j}}(z I-T) .
$$

Let $n$ be a positive integer such that $r_{\sigma}\left(\Delta_{n}\right)<|z|$. Then we have

$$
A_{q}^{(n)}(z)=\left(I-\frac{\Delta_{n}}{z}\right)^{-1}\left(I-\frac{\Delta_{n}^{q}}{z^{q}}\right)(z I-T)
$$

and hence $A_{q}^{(n)}(z)$ is invertible if and only if $z \in \rho(T)$.

Let $\mathrm{T}_{q}^{(n)}$ be the companion matrix of the polynomial $z^{q-1} A_{q}^{(n)}(z)$ :

$$
\left[\begin{array}{ccccc}
T_{n} & \Delta_{n} T_{n} & \cdots & \Delta_{n}^{q-2} T_{n} & \Delta_{n}^{q-1} T \\
I & 0 & \cdots & 0 & 0 \\
0 & \ddots & \ddots & \vdots & \vdots \\
\vdots & & \ddots & 0 & \vdots \\
0 & 0 & \cdots & I & 0
\end{array}\right]
$$

Since the spectrum of a monic operator polynomial is the same as the spectrum of its companion matrix, we see that if $z \in \mathbb{C}$ and $z \neq 0$, then for all large $n, \mathbf{T}_{q}^{(n)}-z I$ is invertible if and only if $z \in \rho(T)$. Note that this matrix differs from the matrix $\mathbf{T}_{q, n}$ only in the last entry of the first row and

$$
\left\|\mathbf{T}_{q}^{(n)}-\mathbf{T}_{q, n}\right\|_{\infty}=\left\|\left(T-T_{n}\right)^{q}\right\|
$$


PROPOSITION 2.2. (a) For all large $n$ and all $q=2,3, \ldots, \Gamma \subset \rho\left(\mathbf{T}_{q}^{(n)}\right)$,

$$
\sigma\left(\mathbf{T}_{q}^{(n)}\right) \cap \operatorname{Int} \Gamma=\sigma(T) \cap \operatorname{Int} \Gamma=\Lambda .
$$

(b) If $\min \{|z|: z \in \Gamma\}>1$, then for all large $n$ and all $q=2,3, \ldots$,

$$
\max _{z \in \Gamma}\left\|\left(\mathbf{T}_{q}^{(n)}-z \mathbf{I}_{q}\right)^{-1}\right\|_{\infty} \leq 2 C,
$$

where $C$ is as in Proposition 2.1 (b).

(c) Let

$$
\mathbf{P}_{q}^{(n)}=-\frac{1}{2 \pi i} \int_{\Gamma}\left(\mathbf{T}_{q}^{(n)}-z \mathbf{I}_{q}\right)^{-1} d z
$$

denote the spectral projections associated with $\mathbf{T}_{q}^{(n)}$ and $\Lambda$. Then $\left\|\mathbf{P}_{q}^{(n)}-\mathbf{P}_{q, n}\right\|_{\infty} \rightarrow 0$ as $n \rightarrow \infty$, uniformly in $q=2,3, \ldots$, so that

$$
\operatorname{rank} \mathbf{P}_{q}^{(n)}=\operatorname{rank} \mathbf{P}_{q, n}=m
$$

for all large $n$ and all $q=2,3, \ldots$

PROOF. (a) Note that $r_{\sigma}\left(\Delta_{n}\right) \leq\left\|\Delta_{n}^{2}\right\|^{1 / 2} \rightarrow 0$ as $n \rightarrow \infty$ by our hypothesis (H). Thus if $n$ is so large that $r_{\sigma}\left(\Delta_{n}\right)<\min \{|z|: z \in \Gamma\}$, then for all $q=2,3, \ldots$, $\Gamma \subset \rho\left(\mathbf{T}_{q}^{(n)}\right)$ and

$$
\sigma\left(\mathbf{T}_{q}^{(n)}\right) \cap \operatorname{Int} \Gamma=\sigma(T) \cap \operatorname{Int} \Gamma=\Lambda .
$$

(b) By Proposition 2.1 (b), we have for all large $n$ and all $q=2,3, \ldots$,

$$
\max _{z \in \Gamma}\left\|\left(\mathbf{T}_{q, n}-z \mathbf{I}_{q}\right)^{-1}\right\|_{\infty} \leq C .
$$

Fix $n_{0}$ such that for $n \geq n_{0}, q=2,3, \ldots$,

$$
\left\|\mathbf{T}_{q}^{(n)}-\mathbf{T}_{q, n}\right\|_{\infty} \leq \frac{1}{2 C}
$$

As a consequence

$$
\max _{z \in \Gamma}\left\|\left(\mathbf{T}_{q}^{(n)}-\mathbf{T}_{q, n}\right)\left(\mathbf{T}_{q, n}-z \mathbf{I}_{q}\right)^{-1}\right\|_{\infty} \leq \frac{1}{2}
$$

Since

$$
\left(\mathbf{T}_{q}^{(n)}-z \mathbf{I}_{q}\right)=\left[\mathbf{I}_{q}-\left(\mathbf{T}_{q, n}-\mathbf{T}_{q}^{(n)}\right)\left(\mathbf{T}_{q, n}-z \mathbf{I}_{q}\right)^{-1}\right]\left(\mathbf{T}_{q, n}-z \mathbf{I}_{q}\right), \quad z \in \Gamma,
$$


it follows that for $n \geq n_{0}, q=2,3, \ldots$,

$$
\max _{z \in \Gamma}\left\|\left(\mathbf{T}_{q}^{(n)}-z \mathbf{I}_{q}\right)^{-1}\right\|_{\infty} \leq \max _{z \in \Gamma} \frac{\left\|\left(\mathbf{T}_{q, n}-z \mathbf{I}_{q}\right)^{-1}\right\|_{\infty}}{1-\left\|\left(\mathbf{T}_{q, n}-\mathbf{T}_{q}^{(n)}\right)\left(\mathbf{T}_{q, n}-z \mathbf{I}_{q}\right)^{-1}\right\|_{\infty}} \leq 2 C .
$$

(c) We have

$$
\begin{aligned}
\left\|\mathbf{P}_{q}^{(n)}-\mathbf{P}_{q, n}\right\|_{\infty} & =\left\|-\frac{1}{2 \pi i} \int_{\Gamma}\left(\left(\mathbf{T}_{q}^{(n)}-z \mathbf{I}_{q}\right)^{-1}-\left(\mathbf{T}_{q, n}-z \mathbf{I}_{q}\right)^{-1}\right) d z\right\|_{\infty} \\
& \leq \frac{\ell(\Gamma) C^{2}}{\pi}\left\|\mathbf{T}_{q}^{(n)}-\mathbf{T}_{q, n}\right\|_{\infty} \\
& \leq \frac{\ell(\Gamma) C^{2}}{\pi}\left\|\left(T-T_{n}\right)^{q}\right\| \rightarrow 0 \text { as } n \rightarrow \infty .
\end{aligned}
$$

Hence if $\left\|\mathbf{P}_{q}^{(n)}-\mathbf{P}_{q, n}\right\|_{\infty} \leq 1$, then $\operatorname{rank} \mathbf{P}_{q}^{(n)}=\operatorname{rank} \mathbf{P}_{q, n}=n$.

In this paper, we consider the case $m=1$, that is, when $\Lambda$ consists of only one nonzero simple eigenvalue $\lambda$ of $T$. Let $P$ denote the spectral projection associated with $T$ and $\lambda$, and let $R(P)$ denote the range of $P$. Since $m=1$, we have

$$
R(P)=N(T-\lambda I) \quad \text { and } \quad R\left(\mathbf{P}_{q}^{(n)}\right)=N\left(\mathbf{T}_{q}^{(n)}-\lambda \mathbf{I}_{q}\right)
$$

Let $\phi$ be an eigenvector of $T$ corresponding to $\lambda$ and $\Phi_{q}=\left[\phi, \frac{\phi}{\lambda}, \ldots, \frac{\phi}{\lambda^{q-1}}\right]^{t}$. Then using (2.1), we obtain

$$
\begin{aligned}
\mathbf{T}_{q}^{(n)} \Phi_{q} & =\left[\left(\sum_{j=0}^{q-2} \frac{\Delta_{n}^{j} T_{n}}{\lambda^{j}}+\frac{\Delta_{n}^{q-1} T}{\lambda^{q-1}}\right) \phi, \phi, \frac{\phi}{\lambda}, \ldots, \frac{\phi}{\lambda^{q-2}}\right]^{t} \\
& =\left[\sum_{j=0}^{q-1} \frac{\Delta_{n}^{j}}{\lambda^{j}}(T-\lambda I) \phi+\lambda \phi, \phi, \frac{\phi}{\lambda}, \ldots, \frac{\phi}{\lambda^{q-2}}\right]^{t} \\
& =\lambda \Phi_{q} .
\end{aligned}
$$

Hence $R\left(\mathbf{P}_{q}^{(n)}\right)=\operatorname{span}\left\{\Phi_{q}\right\}$. Let $\phi^{*}$ be the unique eigenvector of $T^{*}$ corresponding to $\bar{\lambda}$ such that

$$
\left\langle\phi, \phi^{*}\right\rangle=1
$$

If we define $\Phi_{q}^{*}=\left[\phi^{*}, 0, \ldots, 0\right]^{s}$, then

$$
\left\langle\Phi_{q}, \Phi_{q}^{*}\right\rangle=\left\langle\phi, \phi^{*}\right\rangle=1
$$

Since rank $\mathbf{P}_{q, n}=\operatorname{rank} P=1$ for all large $n$ and $q=2,3, \ldots$, consider the nonzero simple eigenvalue $\lambda_{q, n}$ of $T_{q, n}$ which is near $\lambda$. Let $\Phi_{q, n}$ be an eigenvector of 
$\mathbf{T}_{q, n}$ corresponding to $\lambda_{q, n}$ and $\Phi_{q, n}^{*}$ be the unique eigenvector of $\mathbf{T}_{q, n}^{*}$ corresponding to its eigenvalue $\bar{\lambda}_{q, n}$ such that

$$
\left\langle\Phi_{q, n}, \Phi_{q, n}^{*}\right\rangle=1
$$

Then

$$
\mathbf{P}_{q, n} \mathbf{x}=\left\langle\mathbf{x}, \Phi_{q, n}^{*}\right\rangle \Phi_{q, n}, \quad \mathbf{x} \in \mathbf{X}_{q},
$$

and the reduced resolvent $S_{q, n}$ associated with $\mathbf{T}_{q, n}$ and $\lambda_{q, n}$ is given by

$$
\mathbf{S}_{q, n}=\frac{1}{2 \pi i} \int_{\Gamma} \frac{\left(\mathbf{T}_{q, n}-z \mathbf{I}_{q}\right)^{-1}}{z-\lambda_{q, n}} d z .
$$

The operators $\mathbf{T}_{q, n}, \mathbf{P}_{q, n}$ and $\mathbf{S}_{q, n}$ commute with each other. They satisfy

$$
\left(\mathbf{T}_{q, n}-\lambda_{q, n} \mathbf{I}_{q}\right) \mathbf{S}_{q, n}=\mathbf{I}_{q}-\mathbf{P}_{q, n} \text { and } \mathbf{P}_{q, n} \mathbf{S}_{q, n}=\mathbf{0} .
$$

\section{Fixed slope Newton scheme and its convergence analysis}

In analogy with the ordinary fixed slope Newton scheme, we consider the fixed slope Newton scheme of order $q$ with an initial guess $\Phi_{q, n}$ given by

$$
\left.\begin{array}{l}
\lambda_{q, n}^{(0)}:=\lambda_{q, n}, \quad \Phi_{q, n}^{(0)}:=\Phi_{q, n} \quad \text { and for } j=1,2, \ldots, \\
\lambda_{q, n}^{(j)}:=\left\langle\mathbf{T}_{q}^{(n)} \Phi_{q, n}^{(j-1)}, \Phi_{q, n}^{*}\right\rangle \\
\Phi_{q, n}^{(j)}:=\Phi_{q, n}^{(j-1)}-\mathbf{S}_{q, n}\left[\mathbf{T}_{q}^{(n)} \Phi_{q, n}^{(j-1)}-\lambda_{q, n}^{(j)} \Phi_{q, n}^{(j-1)}\right] .
\end{array}\right\}
$$

Note that for a suitably chosen fixed $n$, the first component of the $j$ th iterate $\Phi_{q, n}^{(j)}$ is supposed to approximate an eigenvector of $T$ corresponding to $\lambda$.

For a fixed $q=2,3, \ldots$, we have

$$
\left\|\mathbf{T}_{q}^{(n)}-\mathbf{T}_{q, n}\right\|_{\infty}=\left\|\left(T-T_{n}\right)^{q}\right\| \rightarrow 0 \quad \text { as } n \rightarrow \infty,
$$

by our hypothesis $(\mathrm{H})$. Hence error estimates for the iterates in the $q$ th order fixed slope Newton scheme can be obtained in the usual manner. Of course, the constants appearing in these error estimates may depend on the order $q$ of the spectral analysis. Further, these error estimates will be valid for all $n \geq n_{0}$, where $n_{0}$ may also depend on $q$. With a view to make these considerations independent of $q$, we note that if $|\lambda|>1$, then by Proposition 2.1, there is an integer $n_{0}$ such that for all $n \geq n_{0}$ and $q=2,3, \ldots$,

$$
\max _{z \in \Gamma}\left\|\left(\mathbf{T}_{q, n}-z \mathbf{I}_{q}\right)^{-1}\right\|_{\infty} \leq C,
$$

for some constant $C$ independent of $q$ and $n$. 
LEMMA 3.1. Let $|\lambda|>1$.

(a) For all large $n$ and all $q=2,3, \ldots$, we have

$$
\begin{aligned}
\left\|\mathbf{P}_{q, n}\right\|_{\infty} & \leq \frac{\ell(\Gamma) C}{2 \pi}, & \left\|\left(\mathbf{T}_{q}^{(n)}-\mathbf{T}_{q, n}\right) \Phi_{q}\right\|_{\infty} & \leq \frac{\|\phi\|}{|\lambda|^{q}}\left\|\Delta_{n}^{q} T\right\|, \\
\left\|\mathbf{S}_{q, n}\right\|_{\infty} & \leq \frac{\ell(\Gamma) C}{\pi \operatorname{dist}(\lambda, \Gamma)}, & \left\|\mathbf{P}_{q, n} \Phi_{q}-\Phi_{q}\right\|_{\infty} & \leq \frac{\ell(\Gamma) C\|\phi\|}{2 \pi \operatorname{dist}(\lambda, \Gamma)} \frac{\left\|\Delta_{n}^{q} T\right\|}{|\lambda|^{q}} .
\end{aligned}
$$

(b) If we define the scalars $M_{q, n}=\left\langle\Phi_{q}, \Phi_{q, n}^{*}\right\rangle$ and $N_{q, n}=\left\langle\Phi_{q, n}, \Phi_{q}^{*}\right\rangle$, then $N_{q, n} M_{q, n} \rightarrow 1$ as $n \rightarrow \infty$, uniformly in $q=2,3, \ldots$

PROOF. Since $\lambda$ is an isolated point of $\sigma(T)$ and $|\lambda|>1$, we can choose a curve $\Gamma$ in $\rho(T)$ isolating $\lambda$ from the rest of $\sigma(T)$ and from 0 such that $\min \{|z|: z \in \Gamma\}>1$. (a) The bound for $\left\|\mathbf{P}_{q, n}\right\|_{\infty}$ is immediate. We have

$$
\begin{aligned}
\left\|\left(\mathbf{T}_{q}^{(n)}-\mathbf{T}_{q, n}\right) \Phi_{q}\right\|_{\infty} & =\left\|\left[\frac{\Delta_{n}^{q} \phi}{\lambda^{q}-1}, 0, \ldots, 0\right]^{t}\right\|_{\infty} \\
& =\left\|\left[\frac{\Delta_{n}^{q} T \phi}{\lambda^{q}}, 0, \ldots, 0\right]^{\iota}\right\|_{\infty} \leq \frac{\|\phi\|}{|\lambda|^{q}}\left\|\Delta_{n}^{q} T\right\| .
\end{aligned}
$$

Since $\Phi_{q} \in R\left(\mathbf{P}_{q}^{(n)}\right)$, we have

$$
\begin{aligned}
\left\|\mathbf{P}_{q, n} \Phi_{q}-\Phi_{q}\right\|_{\infty} & =\left\|\mathbf{P}_{q, n} \Phi_{q}-\mathbf{P}_{q}^{(n)} \Phi_{q}\right\|_{\infty} \\
& \leq \frac{1}{2 \pi}\left\|\int_{\Gamma}\left(\left(\mathbf{T}_{q, n}-z \mathbf{I}_{q}\right)^{-1}-\left(\mathbf{T}_{q}^{(n)}-z \mathbf{I}_{q}\right)^{-1}\right) \Phi_{q} d z\right\|_{\infty} \\
& =\frac{1}{2 \pi}\left\|\int_{\Gamma}\left(\mathbf{T}_{q, n}-z \mathbf{I}_{q}\right)^{-1}\left(\mathbf{T}_{q}^{(n)}-\mathbf{T}_{q, n}\right)\left(\mathbf{T}_{q}^{(n)}-z \mathbf{I}_{q}\right)^{-1} \Phi_{q} d z\right\|_{\infty}
\end{aligned}
$$

Noting that $\left(\mathbf{T}_{q}^{(n)}-z \mathbf{I}_{q}\right)^{-1} \Phi_{q}=\Phi_{q} /(\lambda-z)$ for all $z \in \Gamma$, we obtain the desired result. (b) We have

$$
\begin{aligned}
\left|N_{q, n} M_{q, n}-1\right| & =\left|\left\langle\left(M_{q, n} \Phi_{q, n}-\Phi_{q}\right), \Phi_{q}^{*}\right\rangle\right|=\left|\left\langle\mathbf{P}_{q, n} \Phi_{q}-\Phi_{q}, \Phi_{q}^{*}\right\rangle\right| \\
& \leq\left\|\mathbf{P}_{q, n} \Phi_{q}-\Phi_{q}\right\|_{\infty}\left\|\Phi_{q}^{*}\right\|_{1} \leq \frac{\ell(\Gamma) C\|\phi\|\left\|\phi^{*}\right\|}{2 \pi \operatorname{dist}(\lambda, \Gamma)} \frac{\left\|\Delta_{n}^{q} T\right\|}{|\lambda|^{q}} .
\end{aligned}
$$

If $\left\|\Delta_{n}^{2}\right\| \leq|\lambda|^{2}$, then for all $q=2,3, \ldots$,

$$
\frac{\left\|\Delta_{n}^{q}\right\|}{|\lambda|^{q}} \leq \max \left\{1, \frac{\left\|\Delta_{n}\right\|}{|\lambda|}\right\} \frac{\left\|\Delta_{n}^{2}\right\|}{|\lambda|^{2}} .
$$

Hence by our hypothesis (H), it follows that $N_{q, n} M_{q, n} \rightarrow 1$ as $n \rightarrow \infty$, uniformly in $q$. 
For obtaining the bound for $\left\|\mathbf{S}_{q, n}\right\|_{\infty}$, note that $\lambda_{q, n} \rightarrow \lambda$ as $n \rightarrow \infty$, uniformly in $q=2,3, \ldots$ by $[6$, Theorem 3.6$]$. Hence

$$
\left|\lambda_{q, n}-\lambda\right|<\frac{1}{2} \operatorname{dist}(\lambda, \Gamma)
$$

for all large $n$ and all $q=2,3, \ldots$, so that

$$
\left\|\mathbf{S}_{q, n}\right\|_{\infty} \leq \frac{\ell(\Gamma) C}{2 \pi \operatorname{dist}\left(\lambda_{q, n}, \Gamma\right)} \leq \frac{\ell(\Gamma)}{\pi \operatorname{dist}(\lambda, \Gamma)} .
$$

THEOREM 3.2. Suppose that $|\lambda|>1$ and that an eigenvector $\Phi_{q, n}$ of $\mathbf{T}_{q, n}$ corresponding to $\lambda_{q, n}$ is so chosen that its first component $\phi_{q, n}$ satisfies

$$
0<d \leq\left\|\phi_{q, n}\right\| \leq c
$$

for all large $n$ and all $q=2,3, \ldots$, and some constants $c, d$ independent of $q$ and $n$. Then there is a positive integer $n_{1}$ such that for all $n \geq n_{1}$, all $q \geq 2$ and all $j=0,1, \ldots$,

$$
\left|\lambda-\lambda_{q, n}^{(j)}\right|,\left\|M_{q, n}^{-1} \Phi_{q}-\Phi_{q, n}^{(j)}\right\|_{\infty} \leq \frac{a}{|\lambda|^{q}}\left\|\left(T-T_{n}\right)^{q} T\right\|\left\{b\left\|\left(T-T_{n}\right)^{q}\right\|\right\}^{j},
$$

where $a$ and $b$ are constants, independent of $q, n$ and $j$.

PROOF. By part (b) of Lemma 3.1, there is some positive integer $n_{0}$ such that for all $n \geq n_{0}$ and all $q=2,3, \ldots,\left|N_{q, n} M_{q, n}\right| \geq 1 / 2$. Then $M_{q, n} \neq 0$. Note that $\left\langle M_{q, n}^{-1} \Phi_{q}, \Phi_{q, n}^{*}\right\rangle=1$. First we prove that the sequences $\left(1 /\left|M_{q, n}\right|\right)$ and $\left(\left\|\Phi_{q, n}^{*}\right\|_{1}\right)$ are uniformly bounded in $q$ and $n$. By part (b) of Lemma 3.1,

$$
\frac{1}{\left|M_{q, n}\right|}=\frac{\left|N_{q, n}\right|}{\left|M_{q, n} N_{q, n}\right|} \leq 2\left|\left\langle\phi_{q, n}, \phi^{*}\right\rangle\right| \leq 2\left\|\phi_{q, n}\right\|\left\|\phi^{*}\right\| \leq 2 c\left\|\phi^{*}\right\| .
$$

Since $\left\|\mathbf{P}_{q, n}\right\|_{\infty}=\left\|\Phi_{q, n}\right\|_{\infty}\left\|\Phi_{q, n}^{*}\right\|_{1}$ and $\left\|\Phi_{q, n}\right\|_{\infty} \geq\left\|\phi_{q, n}\right\| \geq d$, we have

$$
\left\|\Phi_{q, n}^{*}\right\|_{1} \leq \frac{\left\|\mathbf{P}_{q, n}\right\|_{\infty}}{d} \leq \frac{\ell(\Gamma) C}{2 \pi d} .
$$

Let us now consider the case $j=0$. Since $\Phi_{q, n}^{(0)}=\Phi_{q, n}=\mathbf{P}_{q, n}\left(M_{q, n}^{-1} \Phi_{q}\right)$, we have

$$
\begin{aligned}
\left\|M_{q, n}^{-1} \Phi_{q}-\Phi_{q, n}^{(0)}\right\|_{\infty} & =\frac{\left\|\Phi_{q}-\mathbf{P}_{q, n} \Phi_{q}\right\|_{\infty}}{\left|M_{q, n}\right|} \\
& \leq \frac{\ell(\Gamma) C\|\phi\|}{2 \pi \operatorname{dist}(\lambda, \Gamma)} \frac{\left\|\left(T-T_{n}\right)^{q} T\right\|}{|\lambda|^{q}} 2 c\left\|\phi^{*}\right\|
\end{aligned}
$$


for all large $n$ and all $q=2,3, \ldots$ by part (a) of Lemma 3.1.

Next, since $\mathbf{T}_{q}^{(n)}\left(M_{q, n}^{-1} \Phi_{q}\right)=\lambda M_{q, n}^{-1} \Phi_{q}$ and $\left\langle M_{q, n}^{-1} \Phi_{q}, \Phi_{q, n}^{*}\right\rangle=1$, we have

$$
\begin{aligned}
\left|\lambda-\lambda_{q, n}^{(0)}\right| & =\left|\lambda-\lambda_{q, n}\right| \\
& =\left\langle\left(\mathbf{T}_{q}^{(n)}-\mathbf{T}_{q, n}\right) M_{q, n}^{-1} \Phi_{q}, \Phi_{q, n}^{*}\right\rangle+\left\langle\mathbf{T}_{q, n}\left(M_{q, n}^{-1} \Phi_{q}-\Phi_{q, n}\right), \Phi_{q, n}^{*}\right\rangle \mid \\
& \leq\left(\left\|\left(\mathbf{T}_{q}^{(n)}-\mathbf{T}_{q, n}\right) M_{q, n}^{-1} \Phi_{q}\right\|_{\infty}+\left\|\mathbf{T}_{q, n}\right\|_{\infty}\left\|M_{q, n}^{-1} \Phi_{q}-\Phi_{q, n}\right\|_{\infty}\right)\left\|\Phi_{q, n}^{*}\right\|_{1} .
\end{aligned}
$$

Now

$$
\left\|\left(\mathbf{T}_{q}^{(n)}-\mathbf{T}_{q, n}\right) M_{q, n}^{-1} \Phi_{q}\right\|_{\infty}=\left\|\left[\frac{\Delta_{n}^{q} T \phi}{\lambda^{q} M_{q, n}}, 0, \ldots, 0\right]^{t}\right\|_{\infty} \leq \frac{2 c\left\|\phi^{*}\right\|}{|\lambda|^{q}}\left\|\left(T-T_{n}\right)^{q} T\right\|\|\phi\| .
$$

Since the sequences $\left(\left\|\mathbf{T}_{q, n}\right\|_{\infty}\right)$ and $\left(\left\|\Phi_{q, n}^{*}\right\|_{1}\right)$ are bounded uniformly in $q$ and $n$, we see that there is a positive integer $n_{0}$ such that for all $n \geq n_{0}$ and all $q=2,3, \ldots$,

$$
\left|\lambda-\lambda_{q, n}^{(0)}\right|,\left\|M_{q, n}^{-1} \Phi_{q}-\Phi_{q, n}^{(0)}\right\|_{\infty} \leq \frac{a}{|\lambda|^{q}}\left\|\left(T-T_{n}\right)^{q} T\right\|
$$

for some constant $a$, independent of $q$ and $n$.

Since $|\lambda|>1$, we have $\left\|\Phi_{q}\right\|_{\infty}=\|\phi\|$, so that

$$
\frac{\left\|\Phi_{q}\right\|_{\infty}}{\left|M_{q, n}\right|} \leq 2 c\|\phi\|\left\|\phi^{*}\right\|
$$

Also, since the sequences $\left(\left\|\mathbf{S}_{q, n}\right\|_{\infty}\right)$ and $\left(\left\|\Phi_{q, n}^{*}\right\|_{1}\right)$ are bounded uniformly in $q$ and $n$, there is a positive constant $b$ such that

$$
\left\|\Phi_{q, n}^{*}\right\|_{1} \leq b \text { and }\left\|S_{q, n}\right\|_{\infty}\left(1+\left\|M_{q, n}^{-1} \Phi_{q}\right\|_{\infty}\left\|\Phi_{q, n}^{*}\right\|_{1}+2 a\|T\|\right) \leq b
$$

for all large $n$ and all $q=2,3, \ldots$ If $\left\|\left(T-T_{n}\right)^{2}\right\| \leq 1$, then $\left\|\mathbf{T}_{q}^{(n)}-\mathbf{T}_{q, n}\right\|_{\infty}=$ $\left\|\left(T-T_{n}\right)^{q}\right\| \leq \max \left\{1,\left\|T-T_{n}\right\|\right\}\left\|\left(T-T_{n}\right)^{2}\right\| \rightarrow 0$ as $n \rightarrow \infty$. Hence there is a positive integer $n_{1}$ such that $\left\|\left(T-T_{n}\right)^{q}\right\| \leq 1 / b$ for all $n \geq n_{1}$ and all $q=2,3, \ldots$.

Now we prove the error bounds by induction on $j$. If $j=0$, then the result is proved above. Now assuming that the result holds for $j-1$ with $j \geq 1$, we prove it for $j$. We have

$$
\begin{aligned}
\left|\lambda-\lambda_{q, n}^{(j)}\right| & =\left|\left\langle\left(\mathbf{T}_{q}^{(n)}-\mathbf{T}_{q, n}\right)\left(M_{q, n}^{-1} \Phi_{q}-\Phi_{q, n}^{(j-1)}\right), \Phi_{q, n}^{*}\right\rangle\right| \\
& \leq\left\|M_{q, n}^{-1} \Phi_{q}-\Phi_{q, n}^{(j-1)}\right\|_{\infty}\left\|\mathbf{T}_{q}^{(n)}-\mathbf{T}_{q, n}\right\|_{\infty}\left\|\Phi_{q, n}^{*}\right\|_{1} \\
& \leq \frac{a}{|\lambda|^{q}}\left\|\left(T-T_{n}\right)^{q} T\right\|\left(b\left\|\left(T-T_{n}\right)^{q}\right\|\right)^{j-1}\left\|\left(T-T_{n}\right)^{q}\right\| b \\
& =\frac{a}{|\lambda|^{q}}\left\|\left(T-T_{n}\right)^{q} T\right\|\left(b\left\|\left(T-T_{n}\right)^{q}\right\|\right)^{j} .
\end{aligned}
$$


Also,

$$
\begin{aligned}
M_{q, n}^{-1} \Phi_{q}-\Phi_{q, n}^{(j)}= & S_{q, n}\left\{\left(\mathbf{T}_{q, n}-\mathbf{T}_{q}^{(n)}\right)\left(M_{q, n}^{-1} \Phi_{q}-\Phi_{q, n}^{(j-1)}\right)+\left(\lambda-\lambda_{q, n}^{(j)}\right) M_{q, n}^{-1} \Phi_{q}\right. \\
& \left.+\left(\lambda_{q, n}^{(j)}-\lambda_{q, n}\right)\left(M_{q, n}^{-1} \Phi_{q}-\Phi_{q, n}^{(j-1)}\right)\right\} .
\end{aligned}
$$

Writing $\lambda_{q, n}^{(j)}-\lambda_{q, n}=\lambda_{q, n}^{(j)}-\lambda+\lambda-\lambda_{q, n}$ and using $b\left\|\left(T-T_{n}\right)^{q}\right\| \leq 1$, we have

$$
\begin{aligned}
\left|\lambda_{q, n}^{(j)}-\lambda_{q, n}\right| & \leq \frac{a}{|\lambda|^{q}}\left\|\left(T-T_{n}\right)^{q} T\right\|\left(b\left\|\left(T-T_{n}\right)^{q}\right\|\right)^{j}+\frac{a}{|\lambda|^{q}}\left\|\left(T-T_{n}\right)^{q} T\right\| \\
& \leq 2 a\|T\|\left\|\left(T-T_{n}\right)^{q}\right\|
\end{aligned}
$$

and hence

$$
\begin{aligned}
\left\|M_{q, n}^{-1} \Phi_{q}-\Phi_{q, n}^{(j)}\right\|_{\infty} \leq & \left\|M_{q, n}^{-1} \Phi_{q}-\Phi_{q, n}^{(j-1)}\right\|_{\infty}\left\|S_{q, n}\right\|_{\infty}\left(1+\left\|M_{q, n}^{-1} \Phi_{q}\right\|_{\infty}\left\|\Phi_{q, n}^{*}\right\|_{1}\right. \\
& +2 a\|T\|)\left\|\left(T-T_{n}\right)^{q}\right\| \\
& \leq \frac{a}{|\lambda|^{q}}\left\|\left(T-T_{n}\right)^{q} T\right\|\left(b\left\|\left(T-T_{n}\right)^{q}\right\|\right)^{j-1} b\left\|\left(T-T_{n}\right)^{q}\right\| \\
& =\frac{a}{|\lambda|^{q}}\left\|\left(T-T_{n}\right)^{q} T\right\|\left(b\left\|\left(T-T_{n}\right)^{q}\right\|\right)^{j},
\end{aligned}
$$

as desired.

Now we obtain error estimates for the iterates in the $q$ th order fixed slope Newton scheme (3.1) without requiring that $|\lambda|>1$ and in the process, improve the results for the case $|\lambda|>1$ in the sense that as $|\lambda|$ increases, the estimates become sharper.

For this purpose, we consider a particular kind of scaling of the operators $\mathbf{T}_{q}^{(n)}$ and $\mathbf{T}_{q, n}$. Fix a positive scalar $\alpha$ and define $D_{q}: \mathbf{X}_{q} \rightarrow \mathbf{X}_{q}$ by

$$
D_{q}\left[x_{1}, \ldots, x_{q}\right]^{t}=\left[x_{1}, \alpha x_{2}, \ldots, \alpha^{q-1} x_{q}\right]^{t}, \quad\left[x_{1}, \ldots, x_{q}\right]^{t} \in \mathbf{X}_{q} .
$$

Let $\tilde{\mathbf{T}}_{q}^{(n)}$ and $\tilde{\mathbf{T}}_{q, n}$ denote the operators obtained by replacing $T$ and $T_{n}$ by $\alpha T$ and $\alpha T_{n}$ in $\mathbf{T}_{q}^{(n)}$ and $\mathbf{T}_{q, n}$, respectively. Then

$$
\tilde{\mathbf{T}}_{q}^{(n)}=D_{q}^{-1}\left(\alpha \mathbf{T}_{q}^{(n)}\right) D_{q} \quad \text { and } \quad \tilde{\mathbf{T}}_{q, n}=D_{q}^{-1}\left(\alpha \mathbf{T}_{q, n}\right) D_{q} .
$$

The corresponding spectral projections are given by

$$
\tilde{\mathbf{P}}_{q}^{(n)}=D_{q}^{-1} \mathbf{P}_{q}^{(n)} D_{q} \quad \text { and } \quad \tilde{\mathbf{P}}_{q, n}=D_{q}^{-1} \mathbf{P}_{q, n} D_{q} .
$$

Thus, if $\Phi_{q}$ is an eigenvector of $T_{q}^{(n)}$ corresponding to $\lambda$, then $\tilde{\Phi}_{q}=D_{q}^{-1} \Phi_{q}$ is an eigenvector of $\tilde{\mathbf{T}}_{q}^{(n)}$ corresponding to $\alpha \lambda$. Let $\tilde{\Phi}_{q}^{*}=D_{q} \Phi_{q}^{*}$. Then

$$
\left\langle\tilde{\Phi}_{q}, \tilde{\Phi}_{q}^{*}\right\rangle=\left\langle\Phi_{q}, \Phi_{q}^{*}\right\rangle
$$


Similarly, if $\Phi_{q, n}$ is an eigenvector of $\mathbf{T}_{q, n}$ corresponding to its eigenvalue $\lambda_{q, n}$, then $\tilde{\Phi}_{q, n}=D_{q}^{-1} \Phi_{q, n}$ is an eigenvector of $\tilde{\mathbf{T}}_{q, n}$ corresponding to its eigenvalue $\alpha \lambda_{q, n}$, and if $\Phi_{q, n}^{*}$ is an eigenvector of $\mathbf{T}_{q, n}^{*}$ corresponding to its eigenvalue $\bar{\lambda}_{q, n}$, then $\tilde{\Phi}_{q, n}^{*}=$ $D_{q} \Phi_{q, n}^{*}$ is an eigenvector of $\mathbf{T}_{q, n}^{*}$ corresponding to its eigenvalue $\alpha \bar{\lambda}_{q, n}$, and further $\left\langle\tilde{\Phi}_{q, n}, \tilde{\Phi}_{q, n}^{*}\right\rangle=\left\langle\Phi_{q, n}, \Phi_{q, n}^{*}\right\rangle$.

If $\lambda_{q, n}$ is a simple eigenvalue of $\mathbf{T}_{q, n}$, then the reduced resolvent associated with $\tilde{\mathrm{T}}_{q, n}$ and $\alpha \lambda_{q, n}$ is given by

$$
\tilde{\mathbf{S}}_{q, n}=D_{q}^{-1} \frac{\mathbf{S}_{q, n}}{\alpha} D_{q} .
$$

Let $\alpha$ be a positive scalar. Consider the fixed slope Newton scheme corresponding to the scaled operators:

$$
\left.\begin{array}{l}
\tilde{\lambda}_{q, n}^{(0)}:=\alpha \lambda_{q, n}, \quad \tilde{\Phi}_{q, n}^{(0)}:=D_{q}^{-1} \Phi_{q, n}, \quad \tilde{\Phi}_{q, n}^{*}:=D_{q} \Phi_{q, n}^{*}, \quad \text { and for } j=1,2, \ldots, \\
\tilde{\lambda}_{q, n}^{(j)}:=\left\langle\tilde{\mathbf{T}}_{q}^{(n)} \tilde{\Phi}_{q, n}^{(j-1)}, \tilde{\Phi}_{q, n}^{*}\right\rangle, \\
\tilde{\Phi}_{q, n}^{(j)}:=\tilde{\Phi}_{q, n}^{(j-1)}-\tilde{\mathbf{S}}_{q, n}\left[\tilde{\mathbf{T}}_{q}^{(n)} \tilde{\Phi}_{q, n}^{(j-1)}-\tilde{\lambda}_{q, n}^{(j)} \tilde{\Phi}_{q, n}^{(j-1)}\right] .
\end{array}\right\}
$$

LEMMA 3.3. For $j=0,1, \ldots$,

$$
\tilde{\lambda}_{q, n}^{(j)}=\alpha \lambda_{q, n}^{(j)} \quad \text { and } \quad \tilde{\Phi}_{q, n}^{(j)}=D_{q}^{-1} \Phi_{q, n}^{(j)}
$$

In particular, the first component of the scaled iterate $\tilde{\Phi}_{q, n}^{(j)}$ is the same as the first component of the iterate $\Phi_{q, n}^{(j)}, j=0,1, \ldots$

PROOF. We prove the desired result by induction on $j$. The case $j=0$ is a part of the definition. Assume that the result holds for $j$. Then

$$
\begin{aligned}
\tilde{\lambda}_{q, n}^{(j+1)} & =\left\langle\tilde{\mathbf{T}}_{q}^{(n)} \tilde{\Phi}_{q, n}^{(j)}, \tilde{\Phi}_{q, n}^{*}\right\rangle=\left\langle D_{q}^{-1}\left(\alpha \mathbf{T}_{q}^{(n)}\right) D_{q}\left(D_{q}^{-1} \Phi_{q, n}^{(j)}\right), D_{q} \Phi_{q, n}^{*}\right\rangle \\
& =\alpha\left\langle D_{q}^{-1} \mathbf{T}_{q}^{(n)} \Phi_{q, n}^{(j)}, D_{q} \Phi_{q, n}^{*}\right\rangle=\alpha\left\langle\mathbf{T}_{q}^{(n)} \Phi_{q, n}^{(j)}, \Phi_{q, n}^{*}\right\rangle=\alpha \lambda_{q, n}^{(j+1)}
\end{aligned}
$$

Also,

$$
\begin{aligned}
\tilde{\Phi}_{q, n}^{(j+1)} & =\tilde{\Phi}_{q, n}^{(j)}-\tilde{\mathbf{S}}_{q, n}\left[\tilde{\mathbf{T}}_{q}^{(n)} \tilde{\Phi}_{q, n}^{(j)}-\tilde{\lambda}_{q, n}^{(j+1)} \tilde{\Phi}_{q, n}^{(j)}\right] \\
& =D_{q}^{-1}\left\{\Phi_{q, n}^{(j)}-\frac{\mathbf{S}_{q, n}}{\alpha} D_{q}\left[D_{q}^{-1}\left(\alpha \mathbf{T}_{q}^{(n)}\right) D_{q}\left(D_{q}^{-1} \Phi_{q, n}^{(j)}\right)-\alpha \lambda_{q, n}^{(j+1)} D_{q}^{-1} \Phi_{q, n}^{(j)}\right]\right\} \\
& =D_{q}^{-1}\left\{\Phi_{q, n}^{(j)}-\mathbf{S}_{q, n}\left[\mathbf{T}_{q}^{(n)} \Phi_{q, n}^{(j)}-\lambda_{q, n}^{(j+1)} \Phi_{q, n}^{(j)}\right]\right\} \\
& =D_{q}^{-1} \Phi_{q, n}^{(j+1)} .
\end{aligned}
$$

Thus the result holds for $j+1$ and the inductive step is complete. 
As the first component of $D_{q} \mathbf{x}$ is the same as the first component of $\mathbf{x}$ for each $\mathbf{x} \in \mathbf{X}_{q}$, we see that the first components of $\tilde{\Phi}_{q, n}^{(j)}$ and $\Phi_{q, n}^{(j)}$ are the same.

Since the first components of the eigenvector iterates approximate a suitable eigenvector of $T$, we give error estimates for these first components.

THEOREM 3.4. Suppose that $|\lambda|>\epsilon$ and that an eigenvector $\Phi_{q, n}$ of $\mathbf{T}_{q, n}$ corresponding to $\lambda_{q, n}$ is so chosen that its first component $\phi_{q, n}$ satisfies

$$
0<d \leq\left\|\phi_{q, n}\right\| \leq c
$$

for all large $n$, all $q=2,3, \ldots$, and some constants $c, d$ independent of $q$ and $n$. Let $M_{q, n}=\left\langle\Phi_{q}, \Phi_{q, n}^{*}\right\rangle$. Let $\phi_{q, n}^{(j)}$ denote the first component of $\Phi_{q, n}^{(j)}, j=0,1, \ldots$ Then there is a positive integer $n_{1}$ such that for all $n \geq n_{1}$ and all $q=2,3, \ldots$, $j=0,1, \ldots$,

$$
\left|\lambda-\lambda_{q, n}^{(j)}\right|,\left\|M_{q, n}^{-1} \phi_{q}-\phi_{q, n}^{(j)}\right\| \leq \frac{a^{\prime}}{|\lambda|^{q}}\left\|\left(T-T_{n}\right)^{q} T\right\|\left\{\frac{b^{\prime}}{\epsilon^{q}}\left\|\left(T-T_{n}\right)^{q}\right\|\right\}^{j},
$$

where $a^{\prime}$ and $b^{\prime}$ are constants, independent of $q, n$ and $j$.

Proof. Let $\alpha=1 / \epsilon$. Then $\tilde{\lambda}=\alpha \lambda$ is a simple eigenvalue of $\alpha T$ and $|\tilde{\lambda}|>1$. Consider the scaled operators $\tilde{\mathbf{T}}_{q}^{(n)}$ and $\tilde{\mathbf{T}}_{q, n}$. Now $\tilde{\Phi}_{q, n}=D_{q}^{-1} \Phi_{q, n}$ is an eigenvector of $\tilde{\mathbf{T}}_{q, n}$ corresponding to its eigenvalue $\tilde{\lambda}_{q, n}=\alpha \lambda_{q, n}$. Let $\tilde{\lambda}_{q, n}^{(j)}$ and $\tilde{\Phi}_{q, n}^{(j)}$ be the iterates obtained from (3.2). Note that the first components of $\tilde{\Phi}_{q, n}$ and $\Phi_{q, n}$ are the same. Hence by Theorem 3.2, there is a positive integer $n_{1}$ such that for $n \geq n_{1}$ and all $q=2,3, \ldots, j=0,1, \ldots$,

$$
\begin{aligned}
& \left|\tilde{\lambda}-\tilde{\lambda}_{q, n}^{(j)}\right|,\left\|D_{q}^{-1}\left(M_{q, n}^{-1} \Phi_{q}\right)-\tilde{\Phi}_{q, n}^{(j)}\right\|_{\infty} \\
& \quad \leq \frac{\tilde{a}}{|\tilde{\lambda}|^{q}}\left\|\left(\alpha T-\alpha T_{n}\right)^{q} \alpha T\right\|\left\{\tilde{b}\left\|\left(\alpha T-\alpha T_{n}\right)^{q}\right\|\right\}^{j},
\end{aligned}
$$

where $\tilde{a}$ and $\tilde{b}$ are constants, independent of $q, n$ and $j$. Since

$$
\tilde{\lambda}=\alpha \lambda, \quad \tilde{\lambda}_{q, n}^{(j)}=\alpha \lambda_{q, n}^{(j)} \quad \text { and } \quad \tilde{\Phi}_{q, n}^{(j)}=D_{q}^{-1} \Phi_{q, n}^{(j)}
$$

by Lemma 3.3, and since $\left\|M_{q, n}^{-1} \phi_{q}-\phi_{q, n}\right\| \leq\left\|D_{q}^{-1}\left(M_{q, n}^{-1} \Phi_{q}-\Phi_{q, n}^{(j)}\right)\right\|_{\infty}$, we have

$$
\left|\lambda-\lambda_{q, n}^{(j)}\right| \leq \frac{\tilde{a}}{|\lambda|^{q}}\left\|\left(T-T_{n}\right)^{q} T\right\|\left\{\frac{\tilde{b}}{\epsilon^{q}}\left\|\left(T-T_{n}\right)^{q}\right\|\right\}^{j}
$$

and

$$
\left\|M_{q, n}^{-1} \phi_{q}-\phi_{q, n}^{(j)}\right\| \leq \frac{\alpha \tilde{a}}{|\lambda|^{q}}\left\|\left(T-T_{n}\right)^{q} T\right\|\left\{\frac{\tilde{b}}{\epsilon^{q}}\left\|\left(T-T_{n}\right)^{q}\right\|\right\}^{j} .
$$

Taking $a^{\prime}=\max \{\tilde{a}, \alpha \tilde{a}\}$ and $b^{\prime}=\tilde{b}$, the desired result follows. 
Since the simple eigenvalue $\lambda$ of $T$ is assumed to be nonzero, it is natural to expect that a lower bound $\epsilon>0$ for $|\lambda|$ is available. The preceding Theorem 3.4 then gives a computable estimate

$$
\frac{a^{\prime}}{\epsilon^{q}}\left\|(T-T)^{q} T\right\|\left(\frac{b^{\prime}}{\epsilon^{q}}\left\|\left(T-T_{n}\right)^{q}\right\|\right)^{j}
$$

for the errors in the $j$ th eigenvalue and eigenvector iterates. Of course, if a better lower bound $\epsilon^{\prime}$ for $|\lambda|$ is available, that is, if $0<\epsilon<\epsilon^{\prime}<|\lambda|$, then the preceding error estimate with $\epsilon$ replaced by $\epsilon^{\prime}$ becomes sharper.

\section{Implementation}

In this section we show how the refinement scheme (3.1) can be implemented when each $T_{n}$ is a bounded operator of finite rank. We also show how an eigenvector $\Phi_{q, n}$ of $\mathbf{T}_{q, n}$ can be chosen so that its first component $\phi_{q, n}$ is bounded and also bounded away from zero as required in Theorem 3.4:

For simplicity, we assume that rank $T_{n} \leq n$, so that there are $x_{n, 1}, \ldots, x_{n, n}$ in $X$. and $x_{n, 1}^{*}, \ldots, x_{n, n}^{*}$ in $X^{*}$ such that

$$
T_{n} x=\left\langle x, x_{n, 1}^{*}\right\rangle x_{n, 1}+\cdots+\left\langle x, x_{n, n}^{*}\right\rangle x_{n, n}, \quad x \in X .
$$

We describe a canonical procedure for discretizing $T_{n}$.

Define $F_{n}: X \rightarrow \mathbb{C}^{n}$ and $G_{n}: \mathbb{C}^{n} \rightarrow X$ by

$$
\begin{aligned}
F_{n} x & =\left[\left\langle x, x_{n, 1}^{*}\right\rangle, \ldots,\left\langle x, x_{n, n}^{*}\right)\right]^{t}, & & x \in X, \\
G_{n} u & =u(1) x_{n, 1}+\cdots+u(n) x_{n, n}, & & u=[u(1), \ldots, u(n)]^{t} \in \mathbb{C}^{n},
\end{aligned}
$$

where the superscript $t$ denotes transpose. Then $F_{n}^{*}: \mathbb{C}^{n} \rightarrow X^{*}$ and $G_{n}^{*}: X^{*} \rightarrow \mathbb{C}^{n}$ are given by

$$
\begin{aligned}
F_{n}^{*} v & =v(1) x_{n, 1}^{*}+\cdots+v(n) x_{n, n}^{*}, \quad v=[v(1), \ldots, v(n)]^{t} \in \mathbb{C}^{n}, \\
G_{n}^{*} x^{*} & =\left[\left\langle x^{*}, x_{n, 1}\right\rangle, \ldots,\left\langle x^{*}, x_{n, n}\right\rangle\right]^{t}, \quad x^{*} \in X^{*} .
\end{aligned}
$$

It is easy to see that $G_{n} F_{n}=T_{n}$ and $T_{n}^{*}=F_{n}^{*} G_{n}^{*}$.

Note that for $k=0, \ldots, q-1$, the $(i, j)$ th entry of the $n \times n$ matrix $F_{n} \Delta_{n}^{k} G_{n}$ is $\left(\Delta_{n}^{k} x_{n, j}, x_{n, i}^{*}\right), i, j=1, \ldots, n$. Let

$$
\mathbf{A}_{q, n}=\left[\begin{array}{ccccc}
F_{n} G_{n} & F_{n} \Delta_{n} G_{n} & \cdots & F_{n} \Delta_{n}^{q-2} G_{n} & F_{n} \Delta_{n}^{q-1} G_{n} \\
I_{n} & 0 & \cdots & 0 & 0 \\
0 & \ddots & \ddots & \vdots & \vdots \\
\vdots & & \ddots & 0 & \vdots \\
0 & 0 & \cdots & I_{n} & 0
\end{array}\right],
$$


where $I_{n}$ is the $n \times n$ identity matrix.

The following results can be easily verified.

Proposition 4.1. Let $\mu \in \mathbb{C}, \mu \neq 0$. Then

(i) $\mathbf{T}_{q, n} \mathbf{x}=\mu \mathbf{x}$ for $\mathbf{x} \in \mathbf{X}_{q}$ if and only if $\mathbf{x}=\left[\begin{array}{c}x \\ x / \mu \\ \vdots \\ x / \mu^{q-1}\end{array}\right]$ and

$$
\sum_{j=0}^{q-1} \mu^{-j} \Delta_{n}^{j} T_{n} x=\mu x .
$$

(ii) $\mathbf{A}_{q, n} U=\mu U$ for $U \in \mathbb{C}^{n q}$ if and only if $U=\left[\begin{array}{c}u \\ u / \mu \\ \vdots \\ u / \mu^{q-1}\end{array}\right]$ and

$$
\sum_{j=0}^{q-1} \mu^{-j} F_{n} \Delta_{n}^{j} G_{n} u=\mu u
$$

(iii) If $\mu$ and $x$ satisfy (4.1), then $\mu$ and $F_{n} x$ satisfy (4.2). Conversely, if $\mu$ and $u$ satisfy (4.2), then $\mu$ and $\sum_{j=0}^{q-1} \mu^{-j} \Delta_{n}^{j} G_{n} u$ satisfy (4.1).

Thus $\mu$ is an eigenvalue of $\mathbf{T}_{q, n}$ if and only if $\mu$ is an eigenvalue of $\mathbf{A}_{q, n}$.

Recall that $\lambda_{q, n}$ is the unique nonzero simple eigenvalue of $\mathbf{T}_{q, n}$ near the given nonzero simple eigenvalue $\lambda$ of $T$. Then $\lambda_{q, n}$ is also an eigenvalue of $\mathbf{A}_{q, n}$ near $\lambda$. (In fact, $\lambda_{q, n}$ is a simple eigenvalue of $\mathbf{A}_{q, n}$.) Let $U_{q, n}$ be an eigenvector of $\mathbf{A}_{q, n}$ corresponding to $\lambda_{q, n}$ and $V_{q, n}$ be the eigenvector of $\mathbf{A}_{q, n}^{H}$ corresponding to $\bar{\lambda}_{q, n}$ such that

$$
\left\langle U_{q, n}, V_{q, n}\right\rangle=V_{q, n}^{H} U_{q, n}=1 .
$$

Here the superscript $H$ denotes conjugate transpose. Then

$$
U_{q, n}=\left[u_{q, n}, \frac{u_{q, n}}{\lambda_{q, n}}, \ldots, \frac{u_{q, n}}{\lambda_{q, n}^{q-1}}\right]^{t},
$$

for some nonzero $u_{q, n} \in \mathbb{C}^{n}$. Let

$$
\phi_{q, n}=\sum_{j=0}^{q-1} \lambda_{q, n}^{-j-1} \Delta_{n}^{j} G_{n} u_{q, n} .
$$


Then

$$
\Phi_{q, n}=\left[\phi_{q, n}, \frac{\phi_{q, n}}{\lambda_{q, n}}, \ldots, \frac{\phi_{q, n}}{\lambda_{q, n}^{q-1}}\right]^{t}
$$

is an eigenvector of $\mathbf{T}_{q, n}$ corresponding to $\lambda_{q, n}$. Define $\mathbf{F}_{q, n}: \mathbf{X}_{q} \rightarrow \mathbb{C}^{n q}$ by

$$
\mathbf{F}_{q, n}\left[\begin{array}{c}
x_{1} \\
\vdots \\
x_{q}
\end{array}\right]=\left[\begin{array}{c}
F_{n} x_{1} \\
\vdots \\
F_{n} x_{q}
\end{array}\right] \quad \text { for }\left[\begin{array}{c}
x_{1} \\
\vdots \\
x_{q}
\end{array}\right] \in \mathbf{X}_{q}
$$

Since $F_{n} \phi_{g, n}=u_{q, n}$, we get

$$
\mathbf{F}_{q, n} \Phi_{q, n}=U_{q, n}
$$

It can be easily checked that $\mathbf{F}_{q, n} \mathbf{T}_{q, n}=\mathbf{A}_{q, n} \mathbf{F}_{q, n}$ and $\mathbf{F}_{q, n}^{*} \mathbf{A}_{q, n}^{H}=\mathbf{T}_{q, n}^{*} \mathbf{F}_{q, n}^{*}$. Let $\Phi_{q, n}^{*}=\mathrm{F}_{q, n}^{*} V_{q, n}$. Then

$$
\mathbf{T}_{q, n}^{*} \Phi_{q, n}^{*}=\mathbf{T}_{q, n}^{*} \mathbf{F}_{q, n}^{*} V_{q, n}=\mathbf{F}_{q, n}^{*} \mathbf{A}_{q, n}^{H} V_{q, n}=\bar{\lambda}_{q, n} \mathbf{F}_{q, n}^{*} V_{q, n}=\bar{\lambda}_{q, n} \Phi_{q, n}^{*}
$$

and

$$
\left\langle\Phi_{q, n}, \Phi_{q, n}^{*}\right\rangle=\left\langle\Phi_{q, n}, \mathbf{F}_{q, n}^{*} V_{q, n}\right\rangle=\left\langle\mathbf{F}_{q, n} \Phi_{q, n}, V_{q, n}\right\rangle=\left\langle U_{q, n}, V_{q, n}\right\rangle=1
$$

Let \|\|$_{n}$ be a norm on $\mathbb{C}^{n}$. We assume that

$$
\left\|F_{n}\right\| \leq \alpha \quad \text { and } \quad\left\|G_{n}\right\| \leq \beta
$$

for some constants $\alpha$ and $\beta$, independent of $n$. We remark that the boundedness of the sequences $\left(\left\|F_{n}\right\|\right)$ and $\left(\left\|G_{n}\right\|\right)$ depends on the choice of a norm \|\|$_{n}$ on $\mathbb{C}^{n}$ for each $n=1,2, \ldots$ and, in general, this choice will be dictated by the given norm \|\| on the Banach space $X$. Several examples of commonly used finite rank operators and appropriate choices of a norm \|\|$_{n}$ on $\mathbb{C}^{n}$ are given in [5].

We normalize $u_{q, n}$ such that $\left\|u_{q, n}\right\|_{n}=1$. Let $\left|\lambda_{q, n}\right|>\epsilon>0$. Choose $n$ so large that $\left\|\Delta_{n}^{2}\right\|<\epsilon^{2}$. Then

$$
\begin{aligned}
\left\|\phi_{q, n}\right\| & =\left\|\sum_{j=0}^{q-1} \lambda_{q, n}^{-j-1}\left(\Delta_{n}^{j} G_{n} u_{q, n}\right)\right\| \\
& \leq \sum_{j=0}^{q-1} \frac{\left\|\Delta_{n}^{j}\right\|}{\left|\lambda_{q, n}\right|^{j+1}}\left\|G_{n}\right\| \leq \frac{\beta}{\epsilon} \sum_{j=0}^{\infty} \frac{\left\|\Delta_{n}^{j}\right\|}{\epsilon^{j}} \leq \frac{\beta}{\epsilon} \frac{\left(1+\left\|\Delta_{n}\right\| / \epsilon\right)}{\left(1-\left\|\Delta_{n}^{2}\right\| / \epsilon^{2}\right)} .
\end{aligned}
$$


If, in fact, $\left\|\Delta_{n}^{2}\right\|<\epsilon^{2} / 2$ and $\left\|\Delta_{n}\right\| \leq \gamma$, then

$$
\left\|\phi_{q, n}\right\| \leq \frac{2 \beta}{\epsilon}\left(1+\frac{\gamma}{\epsilon}\right) .
$$

Also,

$$
1=\left\|u_{q, n}\right\|_{n}=\left\|F_{n} \phi_{q, n}\right\|_{n} \leq\left\|F_{n}\right\|\left\|\phi_{q, n}\right\| \leq \alpha\left\|\phi_{q, n}\right\| .
$$

Taking $d=1 / \alpha$ and $c=2 \beta(1+\gamma / \epsilon) / \epsilon$, we have

$$
0<d \leq\left\|\phi_{q, n}\right\| \leq c,
$$

which is precisely the condition assumed in Theorem 3.4.

\section{Numerical examples}

We consider the space $X=C([a, b])$ of all complex-valued continuous functions on the interval $[a, b]$ with the sup norm. Let $T$ be an integral operator on $X$ given by

$$
T x(s)=\int_{a}^{b} k(s, t) x(t) d t, \quad x \in X, s \in[a, b],
$$

where the kernel $k(.,$.$) is continuous on [a, b] \times[a, b]$. Note that $T$ is a compact operator on $X$. In actual computations, $T$ is replaced by its Nyström approximation $\tilde{T}$ given by

$$
\tilde{T} x(s)=\sum_{j=1}^{M} w_{j}^{(M)} k\left(s, t_{j}^{(M)}\right) x\left(t_{j}^{(M)}\right), \quad x \in X, s \in[a, b],
$$

where the positive integer $M$ is very large. The nodes $t_{1}^{(M)}, \ldots, t_{M}^{(M)}$ in $[a, b]$ and the weights $w_{1}^{(M)}, \ldots, w_{M}^{(M)}$ in $\mathbb{C}$ are assumed to give a convergent quadrature formula

$$
Q x=\sum_{j=1}^{M} w_{j}^{(M)} x\left(t_{j}^{(M)}\right), \quad x \in X .
$$

For $n \ll M$, we choose nodes $t_{1}^{(n)}, \ldots, t_{n}^{(n)}$ in $[a, b]$. Let $e_{1}^{(n)}, \ldots, e_{n}^{(n)}$ denote the piecewise linear hat functions associated with the nodes $t_{1}^{(n)}, \ldots, t_{n}^{(n)}$ and consider the piecewise linear interpolatory projection given by

$$
\pi_{n} x=\sum_{j=1}^{n} x\left(t_{j}^{(n)}\right) e_{j}^{(n)}, \quad x \in C([0,1]) .
$$


Define $T_{n}=\pi_{n} T$, the projection approximation of $T$. Then $\left\|T-T_{n}\right\| \rightarrow 0$ if the mesh of the partition tends to zero as $n \rightarrow \infty$. We have

$$
\begin{array}{ll}
F_{n} x=\left[T x\left(t_{1}^{(n)}\right), \ldots, T x\left(t_{n}^{(n)}\right)\right]^{t}, & x \in X, \\
G_{n} u=u(1) e_{1}^{(n)}+\cdots+u(n) e_{n}^{(n)}, & u \in \mathbb{C}^{n} .
\end{array}
$$

We consider the sup norm on $\mathbb{C}^{n}$, so that $\left\|F_{n}\right\| \leq\|T\|$ and $\left\|G_{n}\right\| \leq 1$ for all $n$.

Consider the kernel $k_{\eta}(.,):.[0,1] \times[0,1] \rightarrow \mathbb{R}$ given by

$$
k_{\eta}(s, t)= \begin{cases}\eta & \text { if } t \geq s, \\ \eta+s-t & \text { if } s>t,\end{cases}
$$

where $\eta$ is a real parameter. We choose nodes and weights:

$$
t_{i}^{(M)}= \begin{cases}(i-1 / \sqrt{3}) / M & \text { if } i \text { is odd } \\ (i-1+1 / \sqrt{3}) / M & \text { if } i \text { is even }\end{cases}
$$

and $w_{i}^{(M)}=1 / M, i=1, \ldots, M$. These are obtained by the Compound Gauss Two Point Rule on $[0,1]$. For $n \ll M$, let

$$
t_{i}^{(n)}= \begin{cases}(i-1 / \sqrt{3}) / n & \text { if } i \text { is odd } \\ (i-1+1 / \sqrt{3}) / n & \text { if } i \text { is even }\end{cases}
$$

and $w_{i}^{(n)}=1 / n$ for $i=1, \ldots, n$.

This kernel was considered by Dellwo in [9]. The eigenvalue problem for this kernel becomes unstable as $\eta$ approaches 0 . We refer to [9] for a description of the spectrum of $T$ when $\eta$ approaches 0 . While Dellwo had given numerical results for the largest eigenvalue of $T$ and $\eta=-0.66,-0.68,-0.70$, we consider the largest and the second largest eigenvalues of $T$ and $\eta=-0.66$. Like Dellwo, we have taken $M=500$ and $n=5$ when $q=2,3,4$.

Let $\lambda$ denote the largest eigenvalue of $T$, and $\lambda_{q, n}^{(j)}$ and $\Phi_{q, n}^{(j)}$ denote the eigenvalue and eigenvector iterates obtained from the $q$ th order fixed slope Newton scheme (3.1). Let $\phi_{q, n}^{(j)}$ be the first component of $\Phi_{q, n}^{(j)}$. For $j=0,1, \ldots$, let

$$
r_{q, n}^{(j)}=\left\|T \phi_{q, n}^{(j)}-\lambda_{q, n}^{(j)} \phi_{q, n}^{(j)}\right\|_{\infty}
$$

denote the $j$ th residual. Let $\mu$ denote the second largest eigenvalue of $T$. Let $\mu_{q, n}^{(j)}$ and $\hat{r}_{q, n}^{(j)}$ denote the eigenvalue iterate and the residual for the second largest eigenvalue. Our computations were performed on CDC CYBER-180/840 with an accuracy of 15 digits. 
TABLE 5.1. $\eta=-0.66, q=1, n=30(q n=30)$

\begin{tabular}{|c|cc|cc|}
\hline$j$ & $\left|\lambda-\lambda_{q, n}^{(j)}\right|$ & $r_{q, n}^{(j)}$ & $\left|\mu-\mu_{q, n}^{(j)}\right|$ & $\hat{r}_{q, n}^{(j)}$ \\
\hline 0 & $8.45 \times 10^{-5}$ & $2.01 \times 10^{-2}$ & $1.88 \times 10^{-4}$ & $2.06 \times 10^{-2}$ \\
1 & $4.43 \times 10^{-8}$ & $1.36 \times 10^{-5}$ & $6.62 \times 10^{-8}$ & $1.67 \times 10^{-6}$ \\
2 & $4.52 \times 10^{-11}$ & $1.11 \times 10^{-8}$ & $2.83 \times 10^{-11}$ & $1.38 \times 10^{-8}$ \\
3 & $1.42 \times 10^{-14}$ & $1.01 \times 10^{-11}$ & $9.77 \times 10^{-15}$ & $1.92 \times 10^{-12}$ \\
4 & $9.06 \times 10^{-14}$ & $3.29 \times 10^{-13}$ & $3.55 \times 10^{-14}$ & $1.89 \times 10^{-13}$ \\
\hline
\end{tabular}

TABLE 5.2, $\eta=-0.66, q=2, n=5(q n=10)$

\begin{tabular}{|c|cc|cc|}
\hline$j$ & $\left|\lambda-\lambda_{q, n}^{(j)}\right|$ & $r_{q, n}^{(j)}$ & $\left|\mu-\mu_{q, n}^{(j)}\right|$ & $\hat{r}_{q, n}^{(j)}$ \\
\hline 0 & $3.05 \times 10^{-5}$ & $6.72 \times 10^{-4}$ & $1.83 \times 10^{-4}$ & $2.76 \times 10^{-3}$ \\
1 & $1.16 \times 10^{-8}$ & $2.90 \times 10^{-7}$ & $8.09 \times 10^{-8}$ & $2.21 \times 10^{-6}$ \\
2 & $6.11 \times 10^{-12}$ & $1.46 \times 10^{-10}$ & $1.03 \times 10^{-10}$ & $2.17 \times 10^{-9}$ \\
3 & $7.81 \times 10^{-14}$ & $3.81 \times 10^{-13}$ & $8.61 \times 10^{-14}$ & $2.45 \times 10^{-12}$ \\
\hline
\end{tabular}

TABLE 5.3. $\eta=-0.66, q=3, n=5(q n=15)$

\begin{tabular}{|c|cc|cc|}
\hline$j$ & $\left|\lambda-\lambda_{q, n}^{(j)}\right|$ & $r_{q, n}^{(j)}$ & $\left|\mu-\mu_{q, n}^{(j)}\right|$ & $\hat{r}_{q, n}^{(j)}$ \\
\hline 0 & $4.03 \times 10^{-7}$ & $8.95 \times 10^{-6}$ & $5.76 \times 10^{-6}$ & $9.89 \times 10^{-5}$ \\
1 & $2.24 \times 10^{-12}$ & $5.70 \times 10^{-11}$ & $2.64 \times 10^{-10}$ & $5.95 \times 10^{-9}$ \\
2 & $5.32 \times 10^{-14}$ & $2.22 \times 10^{-13}$ & $3.20 \times 10^{-14}$ & $5.90 \times 10^{-13}$ \\
\hline
\end{tabular}

TABLE 5.4. $\eta=-0.66, q=4, n=5(q n=20)$

\begin{tabular}{|c|cc|cc|}
\hline$j$ & $\left|\lambda-\lambda_{q, n}^{(j)}\right|$ & $r_{q, n}^{(j)}$ & $\left|\mu-\mu_{q, n}^{(j)}\right|$ & $\hat{r}_{q, n}^{(j)}$ \\
\hline 0 & $4.47 \times 10^{-9}$ & $1.02 \times 10^{-7}$ & $1.80 \times 10^{-7}$ & $3.11 \times 10^{-6}$ \\
1 & $5.15 \times 10^{-14}$ & $2.17 \times 10^{-13}$ & $4.79 \times 10^{-13}$ & $9.39 \times 10^{-12}$ \\
\hline
\end{tabular}

Our aim is to approximate eigenelements of the $M \times M$ matrix associated with $\tilde{T}$. Assume that an eigenpair $\left(\lambda_{q, n}, U_{q, n}\right)$ of the matrix $\mathbf{A}_{q, n}$ is already found and the $\mathrm{LU}$ decomposition of $\mathbf{A}_{q, n}-\lambda_{q, n} I_{q n}$ is computed. Then using formulae (4.3) and (4.4), we compute $\Phi_{q, n}$ which requires $M q n+M q$ flops. This is the cost of initialization.

The cost of computation of $\lambda_{q, n}^{(j)}$ is $M n+n^{2}+2 n$ flops. For each iteration we also need to solve a system of the type $\left(\mathbf{A}_{q, n}-\lambda_{q, n} I_{q n}\right) \alpha=\beta$. Since the LU decomposition of the associated matrix is available, the solution of the system costs $(q n)^{2}$ flops. Finally, the computation of the eigenvector iterate $\Phi_{q, n}^{(j)}$ needs $M^{2}+M(q n+2 n+2 q+2)+n^{2}$ flops. Thus the total cost per iteration is $M^{2}+M(q n+3 n+2 q+2)+(q n)^{2}+q n+n^{2}+2 n$. As $M$ is large compared to $q n$, the cost per iteration is dominated by the factor $M^{2}$ irrespective of the value of $q$. 
Table 5.1 gives error estimates for the case $q=1$ which is simply the usual fixed slope Newton scheme $([4,8,11,15])$. Note that the eigenvalue problem solved for initialization is of size 30 and in each iteration a linear system of size 30 is solved.

In Tables 5.2-5.4 we give results for $n=5, \eta=-0.66$ and for $q=2,3,4$. The eigenvalue problem solved for initialization for the $q$ th order fixed slope Newton scheme is of the size $q n$. Also, in each iteration a linear system of size $q n$ is solved.

These tables illustrate how higher order refinement schemes can prove to be efficient and economical. We note that the results in these tables compare favourably with the results given by Dellwo [9, Table 3].

\section{References}

[1] M. Ahues and F. Chatelin, "The use of defect correction to refine the eigenelements of compact integral operators", SIAM J. Numer. Anal. 20 (1983) 1087-1093.

[2] M. Ahues, F. d'Almeida, F. Chatelin and M. Telias, "Iterative refinement techniques for the eigenvalue problem of compact integral operators", in Treatment of Integral Equations by Numerical Methods (eds. C. T. H. Baker and G. F. Miller), (Academic Press, London, 1983) 373-386.

[3] M. Ahues, F. d'Almeida and M. Telias, "Iterative refinement for approximate eigenelements of compact operators, R.A.I.R.O.", Numer. Anal. 18 (1984) 125-135.

[4] M. Ahues and M. Telias, "Refinement methods of Newton type for approximate eigenelements of integral operators", SIAM J. Numer. Anal. 23 (1986) 144-159.

[5] R. Alam, R. P. Kulkarni and B. V. Limaye, "Boundedness of adjoint bases of approximate spectral subspaces and of associated block reduced resolvents", Numer. Funct. Anal. Optimiz. 17 (1996) 473-502.

[6] R. Alam, R. P. Kulkarni and B. V. Limaye. "Accelerated spectral approximation", Mathematics of Computation 67 (1998) 1401-1422.

[7] F. Chatelin, "Numerical computation of the eigenelements of linear integral operators by iterations", SIAM J. Numer. Anal. 15 (1978) 1112-1124.

[8] F. Chatelin, Spectral Approximation of Linear Operators (Academic Press, New York, 1983).

[9] D. Dellwo, "Accelerated spectral refinement with application to integral operators", SIAM J. Numer. Anal. 26 (1989) 1184-1193.

[10] D. Dellwo and M. B. Friedman, "Accelerated spectral analysis of compact operators", SIAM J. Numer. Anal. 21 (1984) 1115-1131.

[11] L. N. Deshpande and B. V. Limaye, "A fixed point technique to refine a simple approximate eigenvalue and a corresponding eigenvector", Numer. Funct. Anal. Optimiz. 10 (1989) 909-921.

[12] I. Gohberg, S. Goldberg and M. A. Kaashoek, Classes of Linear Operators, Volume 1 (BirkhäuserVerlag, Berlin, 1990).

[13] I. Gohberg, P. Lancaster and L. Rodman, Matrix Polynomials (Academic Press, New York, 1982).

[14] R. P. Kulkarni and B. V. Limaye, "Solution of a Schrödinger equation by iterative refinement", J. Austral. Math. Soc. (Series B) 32 (1990) 115-132.

[15] B. V. Limaye, "Spectral perturbation and approximation with numerical experiments", Proceedings of the Centre for Mathematical Analysis, Vol. 13 (Australian National University, 1986).

[16] H. Stetter, "The defect correction principle and discretization methods", Numer. Math. 29 (1978) $425-443$. 